\title{
Multiple bars and secular evolution
}

\section{Juntai Shen}

\author{
Shanghai Astronomical Observatory, CAS China, email: jshen@shao.ac.cn
}

\begin{abstract}
Bars are the most important driver of secular evolution. A significant fraction of barred galaxies also harbor small secondary bars. Secondary bars are visible even in nearinfrared images, so they are not just dusty and blue, but stellar features (Erwin \& Sparke 2002). Since they are quite common, secondary bars are probably long-lived stellar features. The random relative orientation of the two bars indicates that they are dynamically decoupled with different pattern speeds (Buta \& Crocker 1993). Corsini et al. (2003) presented conclusive direct kinematic evidence for a decoupled secondary bar in NGC 2950. Dynamically decoupled secondary bars have long been hypothesized to be a mechanism to drive gas past the ILR of primary bars to feed active galactic nuclei (Shlosman et al. 1989). However, the dynamics of secondary bars are still not well understood, and it is still unclear what role secondary bars play in the AGN fueling process.

Numerical simulations offer the best approach to understanding double-barred systems. Decoupled secondary bar in the earlier gaseous simulations only last a short time $(<1 \mathrm{Gyr}$, e.g. Friedli \& Martinet 1993). Orbital studies of double-barred systems discovered a family of loop orbits that may be building blocks of long-lived nuclear stellar bars (Maciejewski \& Sparke 1997, 2000). To complement orbital studies, which are not fully self-consistent, $N$-body simulations are preferred to further our understanding of double-barred systems. Debattista \& Shen (2007) and Shen \& Debattista (2009) managed to form long-lived double-barred systems with purely collisionless simulations, where a pre-existing rotating pseudo-bulge is introduced initially. The shape and size of secondary bars in the models are comparable to observed ones. They found that the rotation of the two bars is not rigid. The amplitude and pattern speed of the secondary bars oscillate as they rotate through their primary counterparts. Although the secondary bar rotates faster than the primary bar in this model, the stellar velocity field in the central region only shows a weakly twisted kinematic minor axis.

Recently more simulations of double-barred galaxies with simpler initial conditions are explored (Du, Shen \& Debattista 2014). We expect that the new models can be used to crosscheck with the kinematic properties of double-barred galaxies from IFU observations such as SAURON and Atlas3D.
\end{abstract}

\section{References}

Buta, R. \& Crocker, D. A. 1993, AJ, 105, 1344

Corsini, E. M., Debattista, V. P., \& Aguerri, J. A. L. 2003, ApJ, 599, L29

Debattista, V. P. \& Shen, J. 2007, ApJ, 654, L127

Du, M., Shen, J., \& Debattista, V. P. 2014, in preparation

Erwin, P. \& Sparke, L. S. 2002, AJ, 124, 65

Friedli, D. \& Martinet, L. 1993, A\& $A, 277,27$

Shen, J. \& Debattista, V. P. 2009, ApJ, 690, 758

Shlosman, I., Frank, J., \& Begelman, M. C. 1989, Nature, 338, 45

Maciejewski, W. \& Sparke, L. S. 1997, ApJL, 484, L117

Maciejewski, W., \& Sparke, L. S. 2000, MNRAS, 313, 745 